\title{
Les systèmes de santé étatiques: une plus-value pour la Suisse?
}

\section{Remo Osterwalder}

Dr, membre du Comité central de la FMH, responsable du département Médecins en libre-pratique

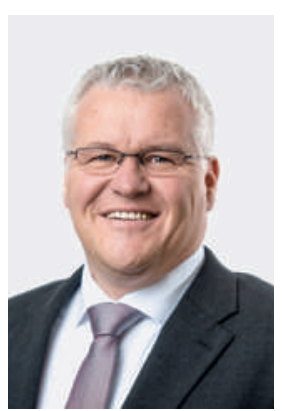

Pour comparer l'efficacité des systèmes de santé, il faut comparer ce qui est comparable. Pour nous, ceci est possible avec des pays comme l'Allemagne, la France, l'Autriche et la Belgique qui connaissent un système décentralisé de médecins de premiers recours et de spécialistes. A l'opposé, on trouve des systèmes qui admettent des médecins de premier recours dans des zones périphériques de manière décentralisée et qui répartissent la quasi-totalité des autres spécialistes dans les hôpitaux. Ces derniers leur délivrent des mandats pour intervenir dans le secteur ambulatoire (Italie, Grèce, Irlande et Pays-Bas). Enfin, troisième variante, il existe des systèmes de soins qui vont encore plus loin et organisent les médecins de premier recours dans des réseaux de soins: c'est le cas de la Finlande, de la Suède, de la Grande-Bretagne, de l'Espagne et du Portugal.

A quels problèmes les systèmes de soins étatiques peuvent-ils être confrontés en Suisse? D'un côté, ils ne sont pas totalement compatibles avec notre système fédéraliste et, de l'autre, ils ne génèrent aucun incitatif en matière d'efficacité. A moyen et long

\section{Avec la médecine d'Etat, le gain d'efficacité se fait au détriment de l'offre et des prestations.}

terme, les coûts ne diminuent pas mais progressent même sensiblement. Ce n'est pas immédiatement perceptible car le secteur ambulatoire est souvent subventionné par l'Etat. Ceci empêche de connaître le décompte des coûts complets, à l'instar de quelques cabinets hospitaliers qui, avec la structure tarifaire TARMED d'aujourd'hui, n'arrivent pas à couvrir leurs dépenses.

\section{Un système de santé étatique permet-il de réaliser des gains d'efficacité?}

Pour répondre à cette question, basons-nous sur les données publiées des médecins de premier recours européens. Alors qu'en Suède et en Norvège un médecin de premier recours traite en moyenne moins de 100 patients par semaine, en Allemagne, ce chiffre est de 250. Avec 100 patients hebdomadaires, la France ne fait pas non plus état d'amélioration de l'efficience. Parti- culièrement élevé, le chiffre allemand s'explique par le système tarifaire actuellement appliqué au domaine ambulatoire. Qu'en est-il des patients [1]? Il n'est pas inintéressant de relever que près de $92 \%$ des personnes interrogées en Allemagne estiment que le praticien leur consacre suffisamment de temps alors qu'en Suède, cette même affirmation ne vaut que pour $74 \%$

La médecine d'Etat ne résout pas les problèmes; elle les déplace.

des cas. En Suisse, une enquête de satisfaction effectuée auprès des consommateurs a clairement montré que les plus de 55 ans se déclarent à $62,4 \%$ très satisfaits du système de santé actuel. En comparaison, ce taux est de 45,6\% pour l'Allemagne, 44,1\% pour la Hollande ou encore de $41 \%$ pour la France [2]. En comparaison européenne, quelle est la satisfaction des médecins au travail? L'Allemagne, la Suède et la France se trouvent tout en bas de l'échelle européenne avec respectivement $54 \%, 75 \%$ et $76 \%$. En se situant à $84 \%$, le degré de satisfaction des médecins suisses est nettement plus élevé [3].

\section{D'où vient cette motivation d'introduire en} Suisse un système de santé dirigé par l'Etat? Le principal intérêt réside dans un contrôle nettement plus marqué que pourraient exercer la Confédération et en partie des cantons, en se voyant attribuer des tâches par délégation en matière d'autorisations de pratiquer et de gestion hospitalière. Cela permettrait certainement d'endiguer les coûts mais réduirait aussi l'accès à des soins du niveau de ceux que nous offrons aujourd'hui. Dans ce contexte, la liberté thérapeutique pour le patient et le médecin n'est plus garantie car aucune réduction des coûts n'est réalisable en maintenant le niveau de soins actuel. Pour trouver des solutions durables et à long terme, il faut réunir tous les partenaires autour d'une table, car ce n'est qu'ensemble que nous pourrons résoudre les problèmes.

1 Statistiques sanitaires de l'OCDE 2013

2 Camenzind P, Petrini L. Les plus de 55 ans dans le système de santé: la Suisse en comparaison internationale. Obsan Dossier 43. Neuchâtel; 2014.

3012 Commonwealth Fund International Health Policy Survey of Primary Care Physicians. 